\title{
AN ANALOG CMOS CIRCUIT FOR COCHLEA IMPLANT
}

\author{
Ying-Chang Chen, Jyh-Horng Chen \\ Department of Electrical Engineering, National Taiwan University, Taipei, Taiwan, R. O. C.
}

\begin{abstract}
The paper presents an analog CMOS circuit simulation of a model for the sensory transduction in the mammalian cochlea. This model consists of basilar membrane, inner hair cell and spiral ganglion of the auditory nerve system. It employs subthreshold CMOS technology and analog circuit which results in a real time, micro power device. Results of its response to sinusoidal excitation simulation is shown.
\end{abstract}

\section{Introduction}

In mammalian cochlea, the acoustic energy present at the ear drum is coupled into a mechanical traveling-wave structure, the basilar membrane, which converts time-domain information into spatially encoded information by spreading out signal in space according to their frequency. At regular interval along the basilar membrane, inner hair cell acts as an electromechanical transducer converting basilar membrane vibration into electrical signals. Several signal processing operations occur during transduction, for example, inner hair cell can half-wave rectify the mechanical signal, responding to motion in only one direction. Inner hair cell has also rapid and short term temporal adaptation properties, as seen in the discharge pattern of auditory nerve fibers; Spiral-ganglion neurons connect to each inner hair cell, and produced fixed width, fixed height pulse in response to inner hair cell electrical activity. Our circuit is designed to produce the basic mechanical and neural behavior of a cochlea.

\section{The model}

The model includes basilar membrane, inner hair cell and spiral ganglion(fig. 1). Input to the model is the time-varying sound pressure pushing upon the oval window of cochlea and the output is the neural firing rate of the individual auditory nerve fibers.

Basilar membrane module: The module in our present model implements the cochlea hydrodynamics using a cascade of 2nd order section with exponentially scaled time constants, which is designed originally by Lyon and Mead[1] and modified to have attenuation at bath sides of cutoff frequency. The analog continuous-time circuit computes the pressure at selected discrete points along the basilar membrane in real time.

Inner hair cell module: The inner hair cell with its attached neurons is an electromechanical transduction system that converts the mechanical motion of the basilar into neural firing activity. In our present model, the inner hair cell module is based on the variable-resistance hair cell model developed by Allen[2]. In the model, the excitation from basilar membrane controls a variable resistance element, and the current through the variable resistor represents the instaneous firing rate on the attached neural fibers. Allen's model has been modified to have two adaptation constants to fit real adaptation data. We have designed an analog CMOS subthreshold circuit to model some aspects of inner hair cell response, including half-wave rectification, and two component adaptation.

Spiral ganglion module: The spiral ganglion neurons connect to each inner hair cell and produce fixed-width, fixed height pulse in response to the inner hair cell. We use the circuit, designed originally by Lazzaro and Mead[3], to create the pulse which is linear to the output current of inner hair cell for modeling the spikes generation of spiral ganglion.

Result

The simulated basilar membrane frequency response of 30 channels with their corresponding cutoff frequency ranged from $200 \mathrm{~Hz}$ to $20 \mathrm{kHz}$ is shown in fig.2. Each curve represents the output of one channel. The temporal characters of adaptation, half-wave rectifying, spikes generation, and steady state response in our inner hair cell and spiral ganglion module are illustrated as the $10 \mathrm{kHz}$ tone-burst response shown in fig.3. The output of inner hair cell is half-wave rectified and is decreased gradually after the tone burst exciting(fig3.(b)). This makes the interval of spikes in spiral ganglion module decrease, too(fig3.(c)).

\section{Discussion}

There is about 4000 transistors in all circuit so that we can include all modules, including basilar membrane, inner hair cell, spiral ganglion, into one chip. The adaptation effect of inner hair cell module should be useful for noise suppression We are working on adding the feedback circuit between inner hair cell and basilar membrane to simulate the function of outer hair cell.

\section{Conclusion}

We have designed a cochlea model for further's cochlea implant applications. The simulation results of our analog CMOS circuit has demonstrated the basic function of mechanical filtering and neural transduction in mammalian 
auditory system. Further improvements of the model are going on to have more functions of mammalian auditory system, for example, the gain-control of outer hair cell. Circuit layout and fabrication of this IC are also under processing.

\section{References}

[1] Mead, C. A., Analog VLSI and Neural Systems. Reading, MA: Addison-Wesley.(1988)

[2] Allen , J. B. , "A hair-cell model of neural response," in Mechanics of Hearing, DeBoer, E. and Viergever, M. A. Eds Hague, The Netherlands, Martinus Nijhoff.(1983)

[3] Lazzaro, J., and Mead, C. A. "Circuit models of sensory transduction in the cochlea," in Analog VLSI Implementation of Neural System, Mead, C. A., and Ismail, M., Eds. Boston, MA: Kluwer Academic Publishers, pp.477-487, May.(1992)

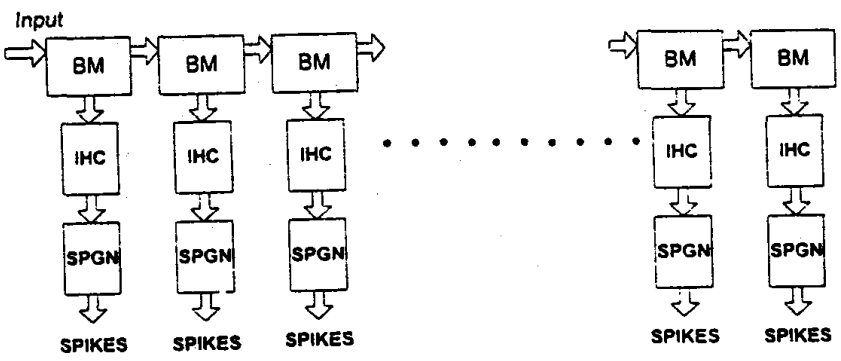

BM: Basilar membrane of cochlear

IHC: Inner hair cell of cochlear

SPGN: Spiral ganglion of auditory nerve

Fig.1 Block diagram of the anaiog CMOS circuit for cochlea

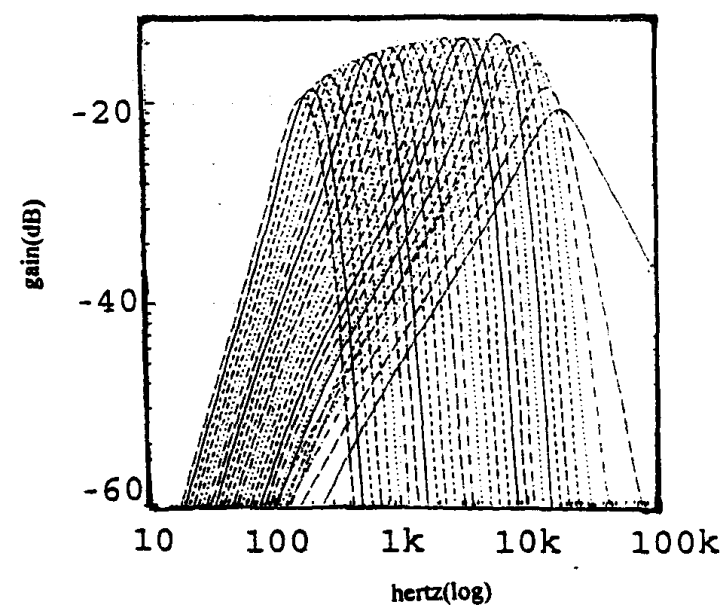

Fig.2 Simulated basilar membrane frequency response

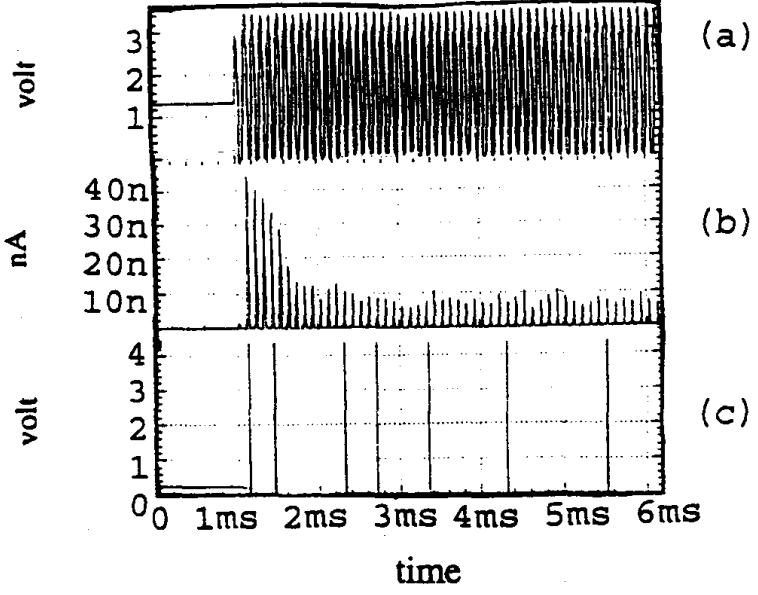

Fig.3 Response of our circuit to pure $10 \mathrm{kHz}$ tone burst

(a) The $10 \mathrm{kHz}$ tone burst input from basilar membrane module.

(b)The simulated output from inner hair cell module

(c) The simulated output from spiral ganglion module 